\title{
Multiclass Combined Models for Urban Travel Forecasting
}

\author{
DAVID BOYCE*,† \\ Department of Civil and Materials Engineering, University of Illinois, Chicago, IL 60607, USA \\ HILLEL BAR-GERA \\ Department of Industrial Engineering and Management, Ben-Gurion University of the Negev, \\ Beer-Sheva 84105, Israel
}

\begin{abstract}
Progress in formulating, solving and implementing models with multiple user classes that combine several travel choices into a single, consistent mathematical formulation is reviewed. Models in which the travel times and costs on the road network are link flow-dependent are considered; such models seek to represent congestion endogenously. The paper briefly summarizes the origins of this field in the 1950s and its evolution through the development of solution algorithms in the 1970s. The primary emphasis of the review is on the implementation and application of multiclass models. The paper concludes with a brief discussion of prospects for improved solution algorithms.
\end{abstract}

Keywords: Transportation network equilibrium, combined travel choice models

\section{Introduction}

To predict travel choices on a congested urban road network, travel times must be endogenous to the model. This statement is axiomatic. Otherwise, the representation of road congestion, a principal causative agent of urban travel and location choices, is implausible. This axiom provided the foundation for the original formulation of the road network equilibrium model by Beckmann et al. (1956). Oddly enough, this seminal contribution, on which the entire field of urban travel choice modeling is implicitly based, was then effectively lost for a decade. By the time it was rediscovered, a sequential, four-step paradigm had taken hold, consisting of: (1) trip generation-to predict the total amount of travel per time period (hour, day) that begins and ends at each location; (2) trip distribution-to predict the amount of travel from every origin to every destination (OD); (3) mode choice-to predict usage of private cars, trains, buses and other modes of travel; and (4) traffic assignment- to predict route choices, in this case user-optimal (UO) choices in which all routes used by travelers have equal travel costs and no unused route has a lower cost for each OD pair (Wardrop, 1952). Modelers then began to ask how to combine these steps into a more

* To whom all correspondence should be addressed.

${ }^{\dagger}$ Current Address: 2149 Grey Avenue, Evanston, IL 60201, USA. 
consistent method, only to arrive at Beckmann et al.'s original formulation. Because of this irony of history, this literature is widely known today as "combined models."

The objective of this paper is to review one type of road network equilibrium model that originated from Beckmann's formulation: multiclass, multimodal, static models of origindestination, mode, departure time period, and route choices, and their relatively recent implementation and validation on large-scale urban transportation systems. Multiclass refers to models that consider two or more classes of travelers with different behavioral or choice characteristics. Multimodal refers to consideration of modes other than the road network with autos and trucks, most commonly some type of public transit system. Static refers to models of constant flows over a congested period, such as the weekday morning or afternoon peak commuting period, possibly divided into intervals of 30 to 60 minutes.

Our focus on this type of models stems from our interest in models that are useful for decision-making about long-range transportation investments, and our conviction that such models have the potential to be substantially superior to current travel forecasting practice. For the best current textbook on travel forecasting, see Ortúzar and Willumsen (2001). The paper begins with a brief historical overview. Next, the principal efforts to implement models are compared and summarized in Table 1 . We conclude with brief observations about the future of this area of research and practice. A reference list emphasizing multiclass model implementations since 1990 completes the review.

\section{Historical overview of combined models}

The historical development of this field, like other scientific pursuits, is complex, in part because separate strands of research have now merged into more comprehensive models. An extensive historical account and mathematically rigorous synthesis of the field with over 1000 references was prepared by Patriksson (1994). Earlier, Sheffi (1985) synthesized his contributions, as well as integrating the findings of other scholars. Oppenheim (1995) set out to write a textbook on travel demand, and succeeded, in addition, to make several theoretical advances to origin-destination-mode choice models based on random utility theory. Syntheses and reviews of combined models were offered by Boyce et al. (1988) and Boyce $(1990,1998)$. Williams (1977) contributed a seminal paper on travel choice models and their implications for evaluation measures, which is highly pertinent to equilibrium modeling.

For the purpose of this review emphasizing model implementation, research is organized by groups of collaborators, or their successors working along a similar line. Beckmann et al. did not follow up on their own accomplishment. Instead, the task of extending Beckmann's model to the multiple-class case was taken up by Dafermos (1972). From her thesis in 1968 until her death in 1990, Dafermos, later in collaboration with Nagurney, established a wideranging theory of traffic equilibrium, including contributions to models with variable as well as fixed demand, treatment of multiple user classes and asymmetric cost functions, and perhaps most importantly extensions and applications of the theory of variational inequalities to transportation network equilibria. See Patriksson (1994) for the list of 18 publications on traffic equilibria by Dafermos too numerous to include in this review. Although highly influential in theoretical and algorithmic developments, neither Dafermos nor Nagurney engaged in model implementation. 
The Centre for Research on Transportation at the University of Montreal, founded in 1972, embarked early on both theoretical research and model implementation and testing. Initially led by Florian, successive generations of faculty and students at Montreal have made sustained contributions to transportation network modeling of several types, including traffic equilibrium. Contributions toward solving the general problem of transportation network equilibrium with variable demand, including mode choice, were made by Florian and Nguyen during the 1970s. Subsequently, many of these methods were implemented in EMME/2, an interactive-graphic multimodal urban transportation planning system developed and distributed by INRO Consultants Inc. The most recent example of this line of research and implementation is a highly detailed model emphasizing transit modes by Florian et al. (2002).

A separate line of research emerged in Great Britain in the mid-1960s. Murchland (1970) sought to devise an alternative to the widely accepted sequential paradigm. Building on his attempt, Evans (1976) examined how to combine trip distribution and traffic assignment models in a single formulation. She succeeded to formulate one version of the combined model as an optimization problem with a convex objective function consisting of two parts, one related to route choice, similar to the objective function in Beckmann's formulation, and the other related to travel demand or trip distribution. In the direct application of the iterative solution method of Frank and Wolfe, each iteration solves a subproblem created by full linearization of the objective function around the current solution, and an averaging of the subproblem solution with the current solution. Evans proposed a partial linearization algorithm, in which only the route choice part of the objective function is linearized in the subproblem.

In a study stimulated by the proposals of Florian and Evans, Frank (1978) compared Evans's partial linearization algorithm with the full linearization approach of Florian for the single-mode, doubly-constrained trip distribution model combined with traffic assignment, and determined that the latter was impractical, even for a 27 zone test problem. Frank's study and research by LeBlanc and Farhangian (1981) were the first implementations of Evans's algorithm.

Boyce initiated an implementation of the Evans formulation and algorithm for the Chicago region in 1979. During the next 15 years he and his students, initially in collaboration with LeBlanc, implemented a single-class, two-mode combined model with 389 zones and about 3,000 road links. Model parameters were first borrowed from other studies, but later estimated in a way that is self-consistent with the model solution (Boyce, 1984; Boyce et al., 1983; Boyce and Zhang, 1998). A textbook chapter synthesizing these developments was offered by Boyce and Daskin (1997).

In 1997, Boyce and Bar-Gera (2001, 2003), with several collaborators, set out to implement, estimate and validate a multiclass, multimodal combined model at the same level of detail used by transportation planning professionals in the Chicago region. The result of this research effort was a three-class model, with provision for expansion to ten classes, estimated on a 1990 household travel survey, and validated on the 1990 Census travel-to-work survey. The model was solved by a generalized Evans algorithm.

In 1986 researchers in Chile began to implement multiclass combined models emphasizing route choices in a congested transit network with several combinations of transit 
modes, as found in Santiago (de Cea et al., 2003). This research led to the development of ESTRAUS and related software, which has been extensively applied to Santiago as well as other Chilean cities. ESTRAUS is currently developed and distributed by Modelos Computacionales de Transporte Limitada. A related research activity was undertaken by Florian et al. (2002), as noted above.

Aashtiani and Magnanti (1981) formulated a combined modal choice and traffic assignment based on nonlinear complementarity theory and studied its solution properties. Later, Safwat and Magnanti (1988) extended this formulation to include trip generation as well as trip distribution, and applied the model to intercity travel forecasting in Egypt and to urban travel in Austin, Texas. Abrahamsson and Lundqvist (1999) substantially extended an earlier study of the Stockholm region to include a parameter estimation method and tested their method on alternative specifications of nested choice functions with a 46 zone, 964 link road network.

\section{Classification of multiclass models}

Simple travel forecasting models assume that all travelers are similar in their travel-decision characteristics, such as their money-value of time and their sensitivity to travel times in choosing their origin, destination and mode of travel, etc. To obtain more realistic models, travelers are often divided into classes, either by socio-economic attributes or by the purpose of their travel (work, shop, etc.), assuming that travel-decision characteristics are the same within each class, but differ among classes.

The introduction of multiple classes increases the mathematical complexity of travel forecasting models. Travel costs in single class models are often separable and symmetric, allowing for convex optimization formulations. In multiclass models travel costs of one class are affected by decisions of other classes; hence the cost structure is not separable, and in general it is not symmetric and does not allow a convex optimization formulation (Altman and Wynter, 2003; Patriksson, 2003). This is indeed the case for some of the multiclass models described in this paper, while in others certain assumptions are made that do allow a convex formulation.

Lam and Huang (1992a) offered a classification of multiclass models, in part based on Abdulaal and LeBlanc (A-L, 1979; L-A, 1982). The following classification corresponds to their classes plus an extension to consider types of classes other than mode-based classes:

1. An O-D matrix is available for each mode. The objective is to obtain the user-optimal (UO) route and link flows, where the link costs are flow-dependent, that is depend on the flows of each mode.

2. The total O-D matrix is known, together with a function of the modal travel costs for allocating each O-D flow to the modes. As above, the objective is to obtain the UO route and link flows, where the link costs are flow-dependent, and there can be mode switching through the mode choice function. As a special case, the mode choice function may allocate all flow for an O-D pair to the lowest cost mode, resulting in UO mode and route flows. 
3. Only the originating and terminating flows for each mode are known, but not the mode's O-D matrix. The objective is to find the modal O-D matrices, such that the route and link flows are UO, where the link costs are flow-dependent.

4. The total originating and terminating flows are known, but not the total O-D matrix, or the O-D matrix by mode. As above, the problem is to find the O-D-mode matrices, and the UO route and link flows, where the link costs are flow-dependent.

5. Finally, as an extension of the above case, the originating and terminating flows are known by classes, such as trip purpose or socio-economic group, but not by mode. As in case 4 , the problem is to determine the O-D-mode flows by class, as well as the UO route and link flows.

Case 1 corresponds to the models studied by Dafermos (1972), as well as van Vliet et al. (1986). Dafermos identified the integrability condition on the objective function that the effects of the classes on each other must be symmetric. For certain problem formulations, such as autos and buses on the same link, this condition appears to be unrealistic.

Case 2 was examined by Florian (1977) and Abdulaal and LeBlanc (1979), as well as LeBlanc and Farhangian (1981). Friesz (1981) found an equivalent optimization formulation that avoids the symmetry restrictions on the link cost functions. However, the resulting mathematical model is not convex and appears to require route enumeration for its solution.

Lam and Huang (1992a, 1992b, 1994) consider case 3. In their model, the symmetry condition on the objective function is satisfied by specifying the generalized cost of travel on link $a$ in terms of travel time, as follows:

$$
c_{a}^{l}\left(f_{a}\right)=t_{a}\left(f_{a}\right)+\sum_{k} x_{a}^{k}\left(b_{k}^{l} / b_{t}^{l}\right)
$$

where

$c_{a}^{l}\left(f_{a}\right)=$ generalized cost of travel on link $a$ by class $l$ (minutes)

$t_{a}\left(f_{a}\right)=$ auto travel time on link $a$ at flow $f_{a}$

$x_{a}^{k}=$ attribute $k$ of link $a$ (length, operating cost, toll, etc.)

$b_{k}^{l}=$ generalized cost parameter of attribute $k$ for class $l$

$b_{t}^{l}=$ value of travel time for class $l$

In their implementation, Lam and Huang consider private auto, truck and franchised bus as the modal-based user classes. They attribute the generalized cost function to van Vliet et al. (1986).

The models of Boyce and his collaborators up to 1997, as well as the models proposed by Evans (1976) and Florian and Nguyen (1978), belong to case 4, with the simplification that the mode flows occur on separate networks defined for each mode. That is, there is no interaction among the mode flows at the route or link level. The models of de Cea and Fernandez (2001) and Florian et al. (2002) belong to case 5. The model of Boyce and BarGera (2003) also belongs to case 5, with the restriction that auto and transit flows occur on separate networks.

The term multiclass was first used by Dafermos, and later by Florian and others, to refer to cases 1, 2 and 3. In this paper, we refer to any model that divides originating and 
terminating flows into classes as a multiclass model, whether or not the flows occur on the same network.

\section{Implementation of multiclass combined models}

In this section, the implementation of four multiclass combined models is described; see Table 1 for a more detailed comparison.

Lam and Huang were evidently the first to describe in the literature a multiclass model with origin-destination as well as route choice. As just noted, their model defines classes in terms of modes, and does not include a mode choice function. Accordingly, origins and destinations must be mode-specific, a serious limitation for a model intended for travel forecasting practice. Autos, trucks and buses do interact on the road network, albeit in a somewhat limited manner: bus flows are pre-specified by link and trucks are evidently converted to auto equivalents. This model was implemented for a portion of the Hong Kong region, and the results compared with a sequential travel forecasting procedure.

de Cea and Fernandez (2001) and de Cea et al. (2003) describe the formulation and solution of ESTRAUS, in many respects the most detailed multiclass, combined model implemented to date. The model is formulated as a variational inequality problem with capacity constraints for all public transit modes, an important consideration for modeling congested systems. Interaction of autos, taxis and buses on the road network are explicitly considered. In addition, metro and exclusive bus lanes are modeled as an independent network. The implementation for Santiago has 13 user classes, 3 trip purposes, 7 pure transit modes and 4 combined modes. The nested logit structure of the O-D-mode choice model has three levels of choice, perhaps the most detailed attempted in an equilibrium framework.

Boyce and Bar-Gera (2001, 2003), with several collaborators, implemented, estimated and validated a detailed model for the Chicago region, that is simpler in structure, but is the largest multiclass, combined model solved to date in terms of the number of zones (1790) and road network size (12,092 nodes; 39,018 links). The O-D-mode choice model is implemented for two user classes; a fixed truck matrix is also assigned to the road network.

A related model was implemented by Marshall and Boyce (Resource Systems Group, 1997). This model has four user classes plus trucks. The O-D choice model is novel in the use of a compound deterrence function consisting of a negative exponential function times a negative power function, both depending on auto generalized travel cost. Mode choices depend on auto vs. transit generalized costs; transit cost is defined as the best transit submode cost at the O-D level of detail. The solution method uses the method of successive averages, since no objective function corresponding to the $\mathrm{O}$-D choice function is available; however, it is based on the Evans algorithm.

All four models reviewed are solved with algorithms based on the partial linearization method proposed by Evans (1976). Parameters of three of the models were estimated from travel survey data and validated in various ways. Taken together, they represent the state of the art of multiclass, combined models intended for travel forecasting practice. 


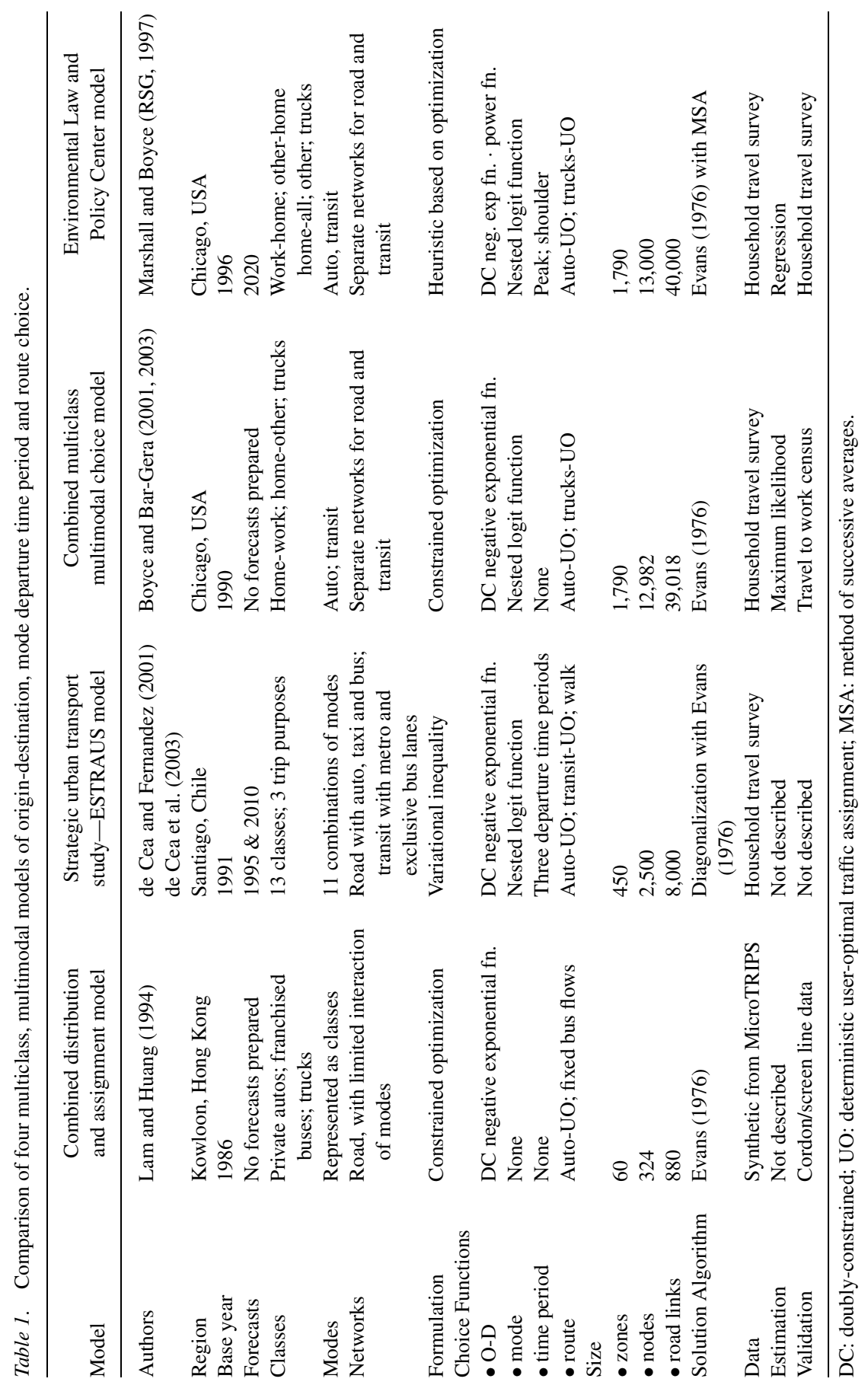




\section{Future prospects}

The implication of using the partial linearization solution method is that the road traffic assignment problem is solved by the full linearization of the sum of the integrals of the link cost functions. As a result, convergence of the solution is relatively slow.

Bar-Gera (2002) introduced the origin-based traffic assignment algorithm, the first algorithm able to solve the traffic assignment problem for large-scale networks to fine convergence with reasonable computing effort. Bar-Gera and Boyce (2003) extended the originbased algorithm to solve a single-class, multimodal combined model, formulated as a fixed point problem. The algorithm rapidly solves this model for the Chicago region road network. Although the algorithm has not yet been applied to a multiclass model, it has the potential of solving a detailed travel forecasting model to the fine convergence necessary for thorough comparisons of alternative land use and transportation scenarios with very reasonable computing effort.

\section{Acknowledgment}

The first author thanks W. H. K. Lam, H. Yang, H. Lo, C. O. Tong, S. C. Wong, H.-J. Huang, H.-P. Lo and J. Dong for fruitful discussions pertaining to combined models and the origin-based traffic assignment algorithm during a visit to Hong Kong and Beijing in 2001.

\section{References}

Aashtiani, H.Z. and T.L. Magnanti. (1981). "Equilibria on a Congested Transportation Network.” SIAM Journal on Algebraic and Discrete Methods 2, 213-226.

Abdulaal, M. and L.J. LeBlanc. (1979). "Methods for Combining Modal Split and Equilibrium Assignment Models." Transportation Science 13, 292-314.

Abrahamsson, T. and L. Lundqvist. (1999). "Formulation and Estimation of Combined Network Equilibrium Models with Applications to Stockholm.” Transportation Science 33, 80-100.

Altman, E. and L. Wynter. (2003). "Equilibrium, Games, and Pricing in Transportation and Telecommunications Networks." Networks and Spatial Economics 4(1), 7-21.

Bar-Gera, H. (2002). "Origin-Based Algorithm for the Traffic Assignment Problem.” Transportation Science 36, 398-417.

Bar-Gera, H. and D. Boyce. (2003). "Origin-Based Algorithms for Combined Travel Forecasting Models." Transportation Research 37B, 405-422.

Beckmann, M., C.B. McGuire, and C.B. Winsten. (1956). Studies in the Economics of Transportation. New Haven: Yale University Press.

Boyce, D.E. (1984). “Network Models in Transportation/Land Use Planning.” In M. Florian (ed.), Transportation Planning Models. Amsterdam: North-Holland, pp. 475-498.

Boyce, D.E. (1990). "Network Equilibrium Models of Urban Location and Travel Choices: A New Research Agenda." In M. Chatterji and R.E. Kuenne (eds.), New Frontiers in Regional Science. New York: Macmillan, pp. 238-256.

Boyce, D. (1998). "Long-Term Advances in the State of the Art of Travel Forecasting Methods." In P. Marcotte and S. Nguyen (eds.), Equilibrium and Advanced Transportation Modelling. Dordrecht: Kluwer, pp. 73-86.

Boyce, D. and H. Bar-Gera. (2001). "Network Equilibrium Models of Travel Choices with Multiple Classes." In M.L. Lahr and R.E. Miller (eds.), Regional Science Perspectives in Economic Analysis. Amsterdam: Elsevier Science, pp. 85-98. 
Boyce, D. and H. Bar-Gera. (2003). "Validation of Urban Travel Forecasting Models Combining OriginDestination, Mode and Route Choices." Journal of Regional Science 43, 517-540.

Boyce, D.E., K.S. Chon, Y.J. Lee, K.T. Lin, and L.J. LeBlanc. (1983). "Implementation and Computational Issues for Combined Models of Location, Destination, Mode and Route Choice." Environment and Planning A 15, 1219-1230.

Boyce, D.E. and M.S. Daskin. (1997). “Urban Transportation.” In C. ReVelle and A. McGarity (eds.), Design and Operation of Civil and Environmental Engineering Systems. New York: Wiley, pp. 277-341.

Boyce, D.E., L.J. LeBlanc, and K.S. Chon. (1988). "Network Equilibrium Models of Urban Location and Travel Choices: A Retrospective Survey,” Journal of Regional Science 28, 159-183.

Boyce, D. and Y. Zhang. (1998). "Parameter Estimation for Combined Travel Choice Models.” In L. Lundqvist, L.-G. Mattsson and T.J. Kim (eds.), Network Infrastructure and the Urban Environment. Berlin: Springer, pp. 177-193.

Dafermos, S.C. (1972). "The Assignment Problem for Multiclass-User Transportation Networks," Transportation Science 6, 73-87.

de Cea, J. and J.E. Fernandez. (2001). "ESTRAUS: A Simultaneous Equilibrium Model to Analyze and Evaluate Multimodal Urban Transportation Systems with Multiple User Classes," In Proceedings of the Ninth World Conference on Transport Research. Seoul, Korea.

de Cea, J., J.E. Fernandez, V. Dekock, A. Soto, and T.L. Friesz. (2003). "ESTRAUS: A Computer Package for Solving Supply-Demand Equilibrium Problems on Multimodal Urban Transportation Networks with Multiple User Classes." Presented at the Annual Meeting of the Transportation Research Board, Washington, D.C.

Evans, S.P. (1976). "Derivation and Analysis of Some Models for Combining Trip Distribution and Assignment." Transportation Research 10, 37-57.

Florian, M. (1977). A Traffic Equilibrium Model of Travel by Car and Public Transit Modes." Transportation Science 11, 166-179.

Florian, M. and S. Nguyen. (1978). "A Combined Trip Distribution, Modal Split and Trip Assignment Model." Transportation Research 12, 241-246.

Florian, M., J.H. Wu, and S. He. (2002). "A Multi-Class Multi-Mode Variable Demand Network Equilibrium Model with Hierarchical Logit Structures. In M. Gendreau and P. Marcotte (eds.), Transportation and Network Analysis: Current Trends. Dordrecht: Kluwer, pp. 119-133.

Frank, C. (1978). "A Study of Alternative Approaches to Combined Trip Distribution-Assignment Modeling." Ph.D. Thesis, Regional Science, University of Pennsylvania, Philadelphia.

Friesz, T.L. (1981). "An Equivalent Optimization Problem for Combined Multiclass Distribution, Assignment and Modal Split which Obviates Symmetry Restrictions." Transportation Research 15B, 361-369.

Lam, W.H.K. and H.-J. Huang. (1992a). "A Combined Trip Distribution and Assignment Model for Multiple User Classes." Transportation Research 26B, 275-287.

Lam, W.H.K. and H.-J. Huang. (1992b). "Calibration of the Combined Trip Distribution and Assignment Model for Multiple User Classes.” Transportation Research 26B, 289-305.

Lam, W.H.K. and H.-J. Huang. (1994). "Comparison of Results of Two Models of Transportation Demand in Hong Kong: CDAM and a Version of MicroTRIPS.” Journal of Advanced Transportation 28, 107-126.

LeBlanc, L.J. and M. Abdulaal. (1982). "Combined Mode Split-Assignment and Distribution-Mode SplitAssignment with Multiple Groups of Travelers.” Transportation Science 16, 430-442.

LeBlanc, L.J. and K. Farhangian. (1981). "Efficient Algorithms for Solving Elastic Demand Traffic Assignment Problems and Mode-Split Assignment Problems." Transportation Science 15, 306-317.

Murchland, J.D. (1970). "Road Network Traffic Distribution in Equilibrium." Mathematical Models in the Social Sciences 8, Meisenheim am Glan, Germany: Anton Hain Verlag, pp. 145-183 (German translation).

Oppenheim, N. (1995). Urban Travel Demand Modeling, New York: Wiley.

Ortúzar, J.D. and L.G. Willumsen. (2001). Modelling Transport, 3rd ed. New York: Wiley.

Patriksson, M. (1994). The Traffic Assignment Problem: Models and Methods. Utrecht: VSP.

Patriksson, M. (2003). "Algorithms for Computing Traffic Equilibria." Networks and Spatial Economics 4(1), 23-38.

Resource Systems Group, Inc. (1997). “Route 53 Alternatives Study, Lake County Model Description,” Environmental Law and Policy Center, Chicago. 
Safwat, K.N.A. and T.L. Magnanti. (1988). “A Combined Trip Generation, Trip Distribution, Modal Split, and Trip Assignment Model," Transportation Science 22, 14-30.

Sheffi, Y. (1985). Urban Transportation Networks. Englewood Cliffs: Prentice-Hall.

van Vliet, D., T. Bergman, and W.H. Scheltes. (1986) "Equilibrium Traffic Assignment with Multiple User Classes," In Proceedings, PTRC Summer Annual Meeting. PTRC Education and Research Services Ltd, London, pp. 111121.

Wardrop, J.G. (1952). "Some Theoretical Aspects of Road Traffic Research." Proceedings of the Institution of Civil Engineers, Part II 1, 325-378.

Williams, H.C.W.L. (1977). "On the Formation of Travel Demand Models and Economic Evaluation Measures of User Benefit.” Environment and Planning A 9, 285-344. 